Experimental verification of sawtooth control by energetic particles in ion cyclotron resonance heated JET tokamak plasmas

This article has been downloaded from IOPscience. Please scroll down to see the full text article.

2010 Nucl. Fusion 50052002

(http://iopscience.iop.org/0029-5515/50/5/052002)

View the table of contents for this issue, or go to the journal homepage for more

Download details:

IP Address: 91.182.158.211

The article was downloaded on 07/05/2010 at 08:36

Please note that terms and conditions apply. 


\title{
LETTER
}

\section{Experimental verification of sawtooth control by energetic particles in ion cyclotron resonance heated JET tokamak plasmas}

\author{
J.P. Graves ${ }^{1}$, I.T. Chapman ${ }^{2}$, S. Coda ${ }^{1}$, T. Johnson ${ }^{3}$, \\ M. Lennholm ${ }^{4}$, B. Alper ${ }^{2}$, M. de Baar ${ }^{5}$, K. Crombe ${ }^{6}$, \\ L.-G. Eriksson ${ }^{7}$, R. Felton ${ }^{2}$, D. Howell ${ }^{2}$, V. Kiptily ${ }^{2}$, \\ H.R. Koslowski ${ }^{8}$, M.-L. Mayoral ${ }^{2}$, I. Monakhov ${ }^{2}$, I. Nunes ${ }^{9}$ and \\ S.D. Pinches ${ }^{2}$ and JET-EFDA Contributors ${ }^{\mathrm{a}}$ \\ JET-EFDA, Culham Science Centre, OX14 3DB, Abingdon, UK \\ ${ }^{1}$ École Polytechnique Fédérale de Lausanne (EPFL), Centre de Recherches en Physique des \\ Plasmas, Association EURATOM-Confédération Suisse, 1015 Lausanne, Switzerland \\ ${ }^{2}$ Euratom/CCFE Fusion Association, Culham Science Centre, Abingdon, UK \\ ${ }^{3}$ Euratom-VR Association, EES, KTH, Stockholm, Sweden \\ ${ }^{4}$ EFDA-JET CSU, Culham Science Centre, Abingdon, OX14 3DB, UK \\ ${ }^{5}$ FOM Instituut voor Plasmafysica Rijnhuizen, Association EURATOM-FOM, The \\ Netherlands \\ ${ }^{6}$ Department of Applied Physics, Ghent University, Rozier 44, 9000 Ghent, Belgium \\ ${ }^{7}$ European Commission, Research Directorate General, B-1049 Brussels, Belgium \\ ${ }^{8}$ Forschungszentrum Jülich $\mathrm{GmbH}$ Institut für Energieforschung - Plasmaphysik 52425 \\ Jülich, Germany \\ ${ }^{9}$ Associação EURATOM/IST, 1049-001, Lisboa, Portugal
}

Received 2 March 2010, accepted for publication 23 April 2010

Published 4 May 2010

Online at stacks.iop.org/NF/50/052002

\begin{abstract}
Experimental evidence from the JET tokamak is presented supporting the predictions of a recent theory (Graves et al 2009 Phys. Rev. Lett. 102 065005) on sawtooth instability control by toroidally propagating ion cyclotron resonance waves. Novel experimental conditions minimized a possible alternate effect of magnetic shear modification by ion cyclotron current drive, and enabled the dependence of the new energetic ion mechanism to be tested over key variables. The results have favourable implications on sawtooth control by ion cyclotron resonance waves in a fusion reactor.
\end{abstract}

PACS numbers: 52.55.Fa, 52.55.Tn, 52.50.Qt, 52.25.Pi, 52.35.Py

(Some figures in this article are in colour only in the electronic version)

Magnetohydrodynamic (MHD) stability of plasmas in the presence of energetic ions is a crucial issue for present and future large tokamak experiments. Such ions include $3.5 \mathrm{MeV}$ fusion alpha particles, and energetic minority ions produced

\footnotetext{
a See the appendix of Romanelli F. et al 2008 Proc. 22nd Int. Fusion Energy Conference (FEC) 2008 (Geneva, Switzerland 2008) (Vienna: IAEA). http://www.iop.org/EJ/article/0029-5515/49/10/104006/nf9_10_104006.pdf
}

by auxiliary heating methods such as neutral beam injection (NBI) and from ion cyclotron resonance frequency (ICRF) waves. Ions trapped within the region of lower magnetic field strength have been shown [1-3] to stabilize an instability known as the sawtooth, located within the core localized $q=1$ rational surface, thereby lengthening the period between sequential plasma relaxations [4]. Without an effective means of shortening the period of sawteeth, the relaxation event can 

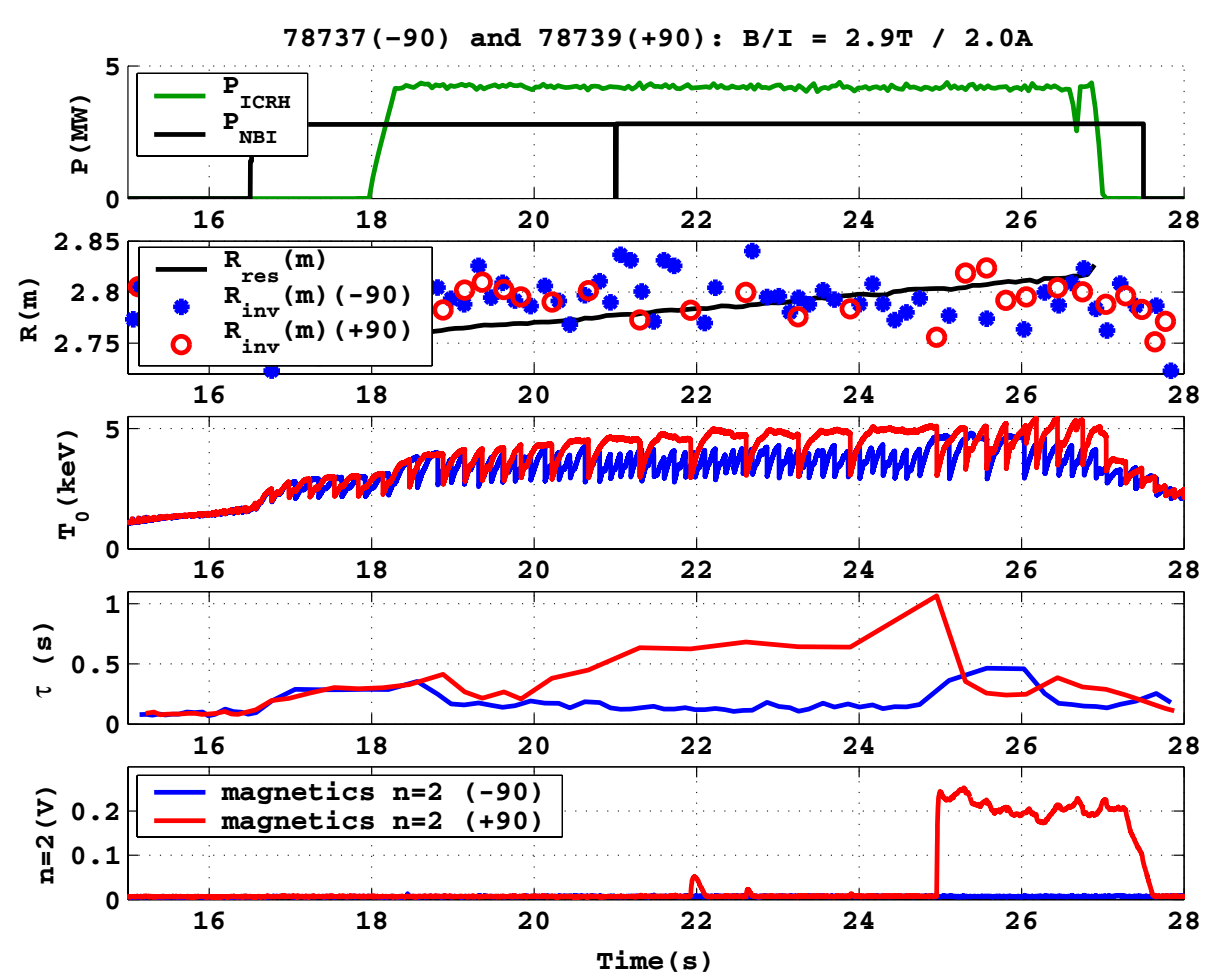

Figure 1. Time traces of NBI and ICRH power, ${ }^{3} \mathrm{He}$ resonance position and inversion radius, central electron temperature, sawtooth period and $n=2$ magnetics amplitude for pulses 78737 (blue, $-90^{\circ}$ phasing) and 78739 (red, $+90^{\circ}$ phasing).

trigger [1,5] secondary performance degrading instabilities located closer to the tokamak edge.

A new explanation was recently given [6] for the highly effective nature of sawtooth control using toroidally propagating ICRF waves with off-axis resonance in tokamaks. Energetic passing ions influence the MHD internal kink mode instability (thought to be responsible for sawteeth) when they are distributed asymmetrically in parallel velocity. Such populations are generated by toroidally aligned NBI, and its effect on sawteeth is well documented [7,8], but parallel velocity asymmetry is also a natural feature of minority ion populations in resonance with toroidally co- or counterpropagating ICRF waves. This letter reports novel dedicated JET experiments which have been devised in order to neutralize an alternative sawtooth control mechanism $[9,10]$ involving changes in the equilibrium current due to ICRF, and permit comparison with recent theory [6] across physical parameters. In the experiments presented here, negligible change to the net equilibrium current was assured by choosing ${ }^{3} \mathrm{He}$ minority ICRF in a deuterium majority plasma $\left({ }^{3} \mathrm{He}\right) \mathrm{D}$, whereby the current dragged $[9,11]$ by the background plasma tends to cancel the ${ }^{3} \mathrm{He}$ current. The experiments reported here are important not least because it had been widely accepted that the poor current drive efficiency arising from minority ${ }^{3} \mathrm{He}$ resonant toroidally propagating waves would not $[9,12]$ provide sawtooth control in deuterium or deuterium-tritium reactor relevant plasmas. Minority ${ }^{3} \mathrm{He}$ experiments prior to [9] had reported results $[1,2]$ with resonance close to the magnetic axis, thereby lengthening sawteeth through the well recognized effect (e.g. [3]) of an axially peaked fast ion pressure. Subsequent sawtooth relevant experiments have, until now, primarily employed high concentration ${ }^{3} \mathrm{He}$ (e.g.
[13] and references therein), leading to mode conversion and direct electron heating close to the $q=1$ surface, and consequently efficient local current profile and sawtooth modification. In contrast, and contrary to the predictions of $[9,12]$ the novel experiments outlined in this letter show that sawtooth control by toroidally propagating ICRF waves, with $q=1$ localized resonance on low concentration minority ${ }^{3} \mathrm{He}$, is in fact extremely effective, as predicted by the fast ion mechanism [6]. This demonstrates the viability of sawtooth control using ICRH in ITER [14], which is primarily expected to employ ${ }^{3} \mathrm{He}$ minority [12].

The effectiveness of minority ${ }^{3} \mathrm{He}$ ICRF for controlling sawteeth, and its importance, is illustrated in figure 1 . The only difference between the two pulses is that the direction of the toroidally propagating ICRF waves is counter-tangent to the plasma current in pulse $78737\left(-90^{\circ}\right.$ antenna phasing) and co-tangent in pulse $78739\left(+90^{\circ}\right.$ antenna phasing). In both pulses the early NBI phase increases the sawtooth period to $300 \mathrm{~ms}$ from Ohmic (without auxiliary heating) sawteeth of around $80 \mathrm{~ms}$. At $18 \mathrm{~s}, 4.5 \mathrm{MW}$ of ${ }^{3} \mathrm{He}$ ICRF is applied on the high field side of the $q=1$ rational surface, indicated by the resonance position and soft-x-ray resolved inversion major radius in figure 1 . The toroidal magnetic field is then ramped very slowly from $B=2.9 \mathrm{~T}$ to $B=2.96 \mathrm{~T}$, whilst changing the current proportionally in order to keep the $q$ profile stationary. It is seen that for $-90^{\circ}$ phasing the sawtooth period is reduced to a minimum of $100 \mathrm{~ms}$, close to that of Ohmic sawteeth, while for $+90^{\circ}$ phasing, the sawteeth become extremely long. The longest sawtooth period is more than $1 \mathrm{~s}$, and the crash triggers a saturated amplitude resistive mode, specifically a neoclassical tearing mode (NTM) [15], as indicated by the $n=2$ toroidal mode number magnetic signal shown in figure 1 . 


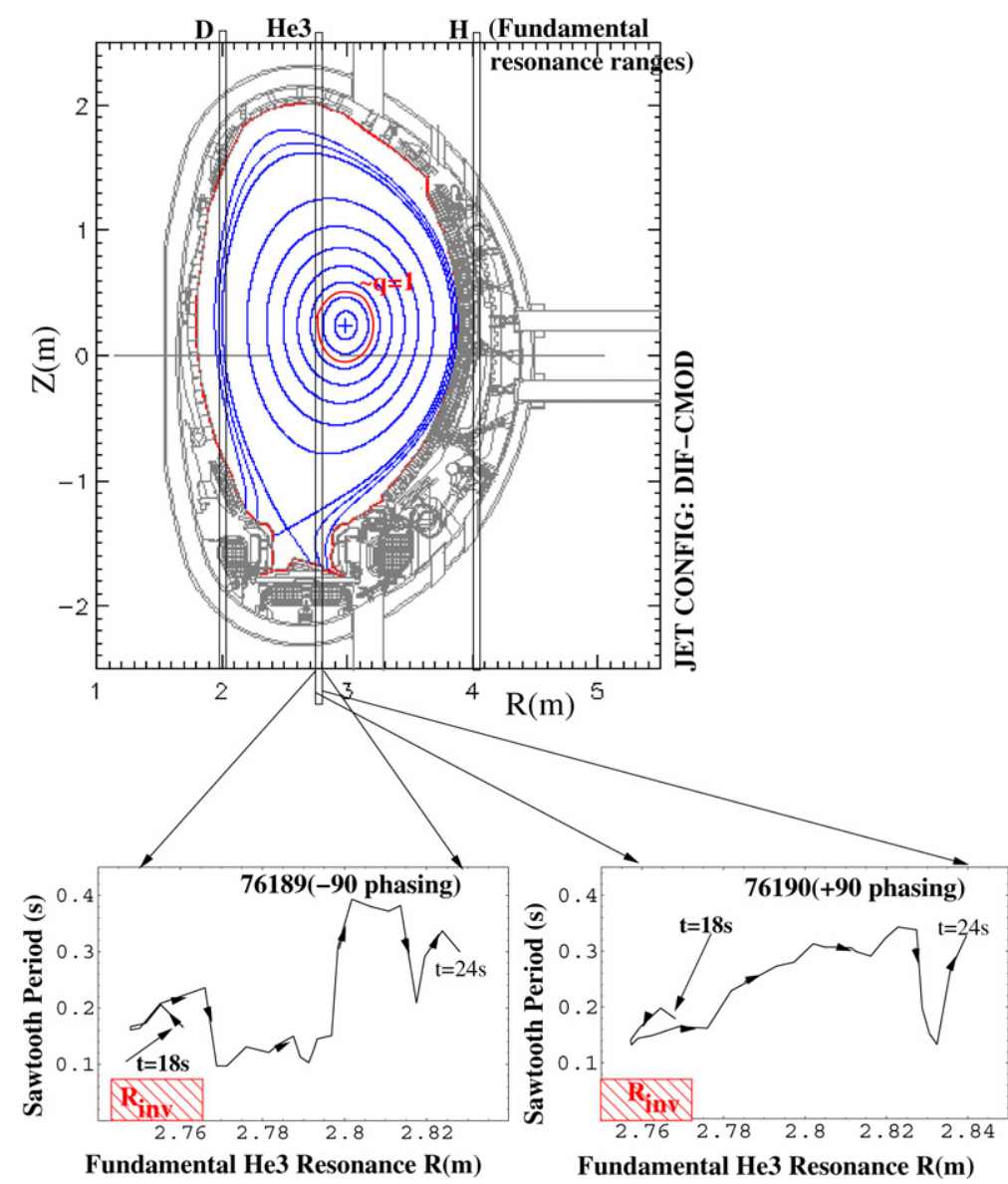

Figure 2. Showing the configuration, and the approximate resonance ranges and locations of ${ }^{3} \mathrm{He}, \mathrm{D}$ and $\mathrm{H}$ over the range of the toroidal magnetic field $2.88 \mathrm{~T}<B<2.96 \mathrm{~T}$. Shown also are the corresponding changes to the sawtooth period in ${ }^{3} \mathrm{He}$ minority pulses $76189\left(-90^{\circ}\right.$ phasing) and $76190\left(+90^{\circ}\right.$ phasing), and the range of the inversion radius given by the shaded box. (Colour online.)

This is a rare observation of an NTM in a low confinement mode plasma with low normalized beta $\left(\beta_{\mathrm{N}} \approx 0.8\right.$, where beta is a figure of merit for a fusion plasma, defined as the ratio of plasma pressure to magnetic pressure), and thus highlights the crucial importance of sawtooth control.

Resolving the mechanism responsible for the sawtooth experiments in JET is very important for predictions of sawtooth control capability in ITER. A widely accepted necessary criterion for instability [16] is given by the kinetic resistive $m=n=1$ internal kink mode threshold:

$$
\frac{\pi \delta \hat{W}}{s_{1}}<\hat{\rho},
$$

where $\delta \hat{W}$ is the potential energy of the internal kink mode (with linear growth rate $\gamma=-\omega_{\mathrm{A}} \pi \delta \hat{W} / s_{1}, \omega_{\mathrm{A}}=v_{\mathrm{A}} / 3^{1 / 2} R_{0}$, $v_{\mathrm{A}}$ the Alfvén velocity and $R_{0}$ the major radius at the magnetic axis), $s_{1}$ is the magnetic shear at the $q=1$ rational surface, where $s=(r / q) \mathrm{d} q / \mathrm{d} r$ and $\hat{\rho}$ is the Larmor radius of the background thermal ions normalized to the $q=1$ minor radius $r_{1}$. In moderate sized present day machines $[17,18]$ convincing evidence exists showing that sawteeth are shortened by increasing $s_{1}$ through localized electron cyclotron current drive (ECCD) techniques such that (1) might be met more rapidly following the previous sawtooth crash. However, in ITER, $\delta \hat{W}$ will typically be very large and positive due to the stabilizing effect of trapped fusion alpha particles, whilst $\hat{\rho}$ will be much smaller than in most present day experiments. Consequently, in ITER, an actuator will have to generate a very large change in $s_{1}$ in order to satisfy (1). By contrast, the fast ion mechanism proposed in [6] generates a change in the macroscopic energy of the internal kink mode due to 'RF' ions, $\delta \hat{W}_{\mathrm{RF}}$, and as a result, it is envisaged that the criterion for instability (e.g. (1)) can be met even when there is a significant stabilizing trapped ion population in the core, and especially in conjunction with enhanced $s_{1}$, via e.g. an additional ECCD actuator. In the JET experiments presented here, a low power NBI ion population plays the role of alpha particles, thus initially lengthening the sawteeth via the the stabilizing contribution $\delta \hat{W}_{\mathrm{NBI}}$ from both trapped fast ions $[1,3]$ and asymmetrically distributed passing ions $[7,8]$. These sawteeth are controlled by the effect [6] of ICRH generated energetic passing ions intersecting the $q=1$ radius, thus reducing, or changing the sign of, the total fast ion contribution $\delta \hat{W}_{\mathrm{RF}}+\delta \hat{W}_{\mathrm{NBI}}$.

It is now shown that sawteeth are modified by ICRH even for pulses with low auxiliary power. Diagnostic neutral beams with a power of 1.4 MW were used in pulses 76189, employing $3 \mathrm{MW}$ of ICRF with $-90^{\circ}$ phasing, and 76190 , employing $2 \mathrm{MW}$ ICRF with $+90^{\circ}$ phasing, both with low concentration (up to $0.5 \%$ ) minority ${ }^{3} \mathrm{He}$. The configuration is essentially the same for all the pulses described here and is shown in 
figure 2. The toroidal magnetic field was ramped upwards from around 2.88 to $2.96 \mathrm{~T}$, and the plasma current was ramped proportionally. Figure 2 plots the sawtooth period for 76189 and 76190 as a function of the ${ }^{3} \mathrm{He}$ resonance position. It is seen that the sawtooth period is strongly modified as the resonance position is shifted relative to the sawtooth inversion radius, shaded in red. The pulse with $-90^{\circ}$ phasing exhibits a narrow window of sawtooth destabilization, while the $+90^{\circ}$ phasing pulse exhibits the opposite.

The experimental objective of generating negligible minority ion current is now addressed. The MSE diagnostic in JET has a typical accuracy of $10 \%$ of the current density $\left(\approx 1.3 \mathrm{MA} \mathrm{m}^{-2}\right.$ at $\left.r_{1}\right)$, which precludes the direct measurement of the ICRF driven current because, as seen below, it is typically two orders of magnitude smaller than the total current density, even in the absence of the plasma drag current. Shown in figure 3 is a SELFO [19] calculation of the fast ion current density $j_{\mathrm{h}}=e Z_{\mathrm{h}} \int \mathrm{d} v^{3} v_{\|} F_{\mathrm{h}}$ for pulse 76189 at $21 \mathrm{~s}$, where $F_{\mathrm{h}}$ is the ICRH distribution function. For the simulation of this pulse, with $-90^{\circ}$ phasing, SELFO employs a spectrum of toroidal wave numbers with maximum power for $n_{\phi}=-15$, while the simulation of $76190\left(+90^{\circ}\right.$ phasing) has maximum power for $n_{\phi}=15$. The asymmetry in toroidal wave number spectra, due to the antenna phasing, gives rise to Fisch currents [11] and currents due to preferential detrapping [20] of co- and counter-circulating ions. SELFO calculates these currents in addition to currents that are insensitive to antenna phasing which arise from the guiding centre drift orbits of predominantly trapped and barely passing ions. However, the plasma is dragged $[9,11]$ along with the fast ions, such that the total current is proportional to a drag coefficient $j_{\mathrm{d}}$, giving $j_{\text {tot }}=j_{\mathrm{h}} \times j_{\mathrm{d}}$. The fast ion current is subject to momentum conservation, quasi-neutrality and the balance of collision rates of electrons on all ion species [9, 11], giving

$$
\begin{aligned}
j_{\mathrm{d}}= & 1-\left[\frac{Z_{\mathrm{h}}}{Z_{\mathrm{eff}}}+\frac{m_{\mathrm{h}} \sum_{i} Z_{i} n_{i}\left(1-Z_{i} / Z_{\text {eff }}\right)}{Z_{h} \sum_{i} n_{i} m_{i}}\right. \\
& \left.-G\left(\frac{Z_{\mathrm{h}}}{Z_{\text {eff }}}-\frac{m_{\mathrm{h}} \sum_{i} n_{i} Z_{i}^{2}}{Z_{\mathrm{h}} Z_{\text {eff }} \sum_{i} n_{i} m_{i}}\right)\right],
\end{aligned}
$$

where $G=1.46 A\left(Z_{\text {eff }}\right) \epsilon^{1 / 2}, A$ is a weak function of $Z_{\text {eff }}$ and $i$ denotes ion species other than hot $(h)$. In figure 3 it is seen that $j_{\mathrm{h}}$ has a dipole structure, with maximum current around $30 \mathrm{kA} \mathrm{m}^{-2}$. Due to the minority ion mass number $m_{\mathrm{h}}=3$ and charge $Z_{\mathrm{h}}=2$, deuterium bulk ion population, carbon and beryllium impurities and moderate $Z_{\text {eff }} \approx 1.8$, the effect of the plasma drag, also shown in figure 3 , is to lower the net driven current density by at least $90 \%$ within the $q=1$ surface, so that the change in the shear $\Delta s$ due to current drive is negligible, as also shown in figure 3 . It is therefore concluded that the sawteeth were not controlled by the effect of ICRF current drive on $s_{1}$. Moreover, that the trend in the sawteeth is opposite for +90 and -90 phasings rules out the possibility that the sawteeth were modified simply by the effect of localized electron heating.

Employing the SELFO generated distribution function for pulses 76189 and 76190 in the drift kinetic code HAGIS [21], together with an MHD displacement supplied from linear ideal
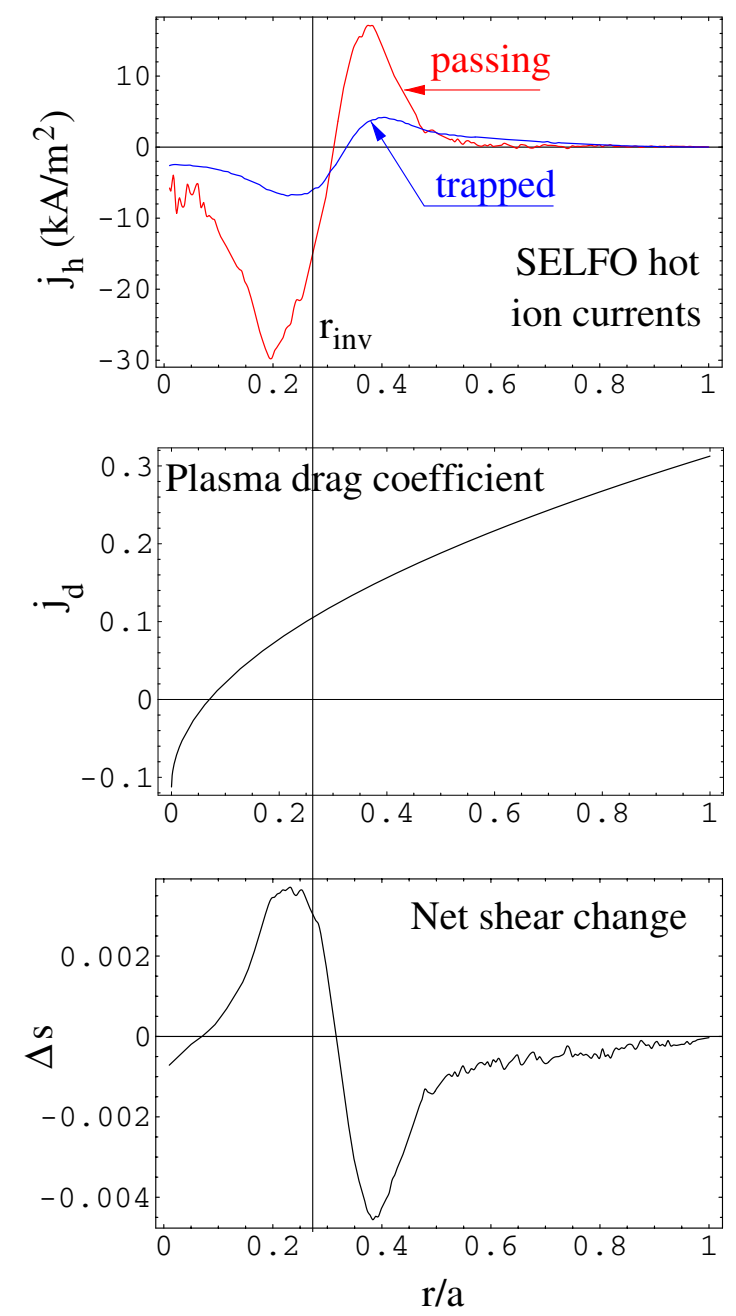

Figure 3. Plotting the passing and trapped contributions to the fast ion current $j_{\mathrm{h}}$ for pulse 76189 , the plasma drag coefficient $j_{\mathrm{d}}$ and change in magnetic shear $\Delta s$. (Colour online.)

MHD numerical calculations, reveals the corresponding fast ion contribution to $\delta \hat{W}$ without recourse to approximation of wave and guiding centre linear interaction. Figure 4 compares the observed signature of the sawtooth period with the fast ion potential energy when plotted with respect to the difference between the ${ }^{3} \mathrm{He}$ resonance position and the measured and averaged inversion radius. The narrow region over which the sawteeth are sensitive to the ICRF deposition, also visible in figure 2 , is recovered by the simulations, which assume $r_{1}=r_{\text {inv }}$. The sign of the RF ion $\delta \hat{W}$ contributions is consistent with the observed effect on the sawteeth, and the amplitude is larger than the resistive threshold $s_{1} \hat{\rho} / \pi$, and all other contributions to $\delta \hat{W}$ including that from the NBI ions. We note from figure 4 that the response of the trapped ions is dwarfed by the passing ion response, as expected from the mechanism described in [6].

By exploiting the knowledge of the fast ion control mechanism derived in [6], it has been possible to reduce its effect, and the corresponding sawtooth control, thereby providing further experimental evidence in support of the theory. The aim is to reduce the finite orbit width of the fast ions, which scales with the hot ion temperature 

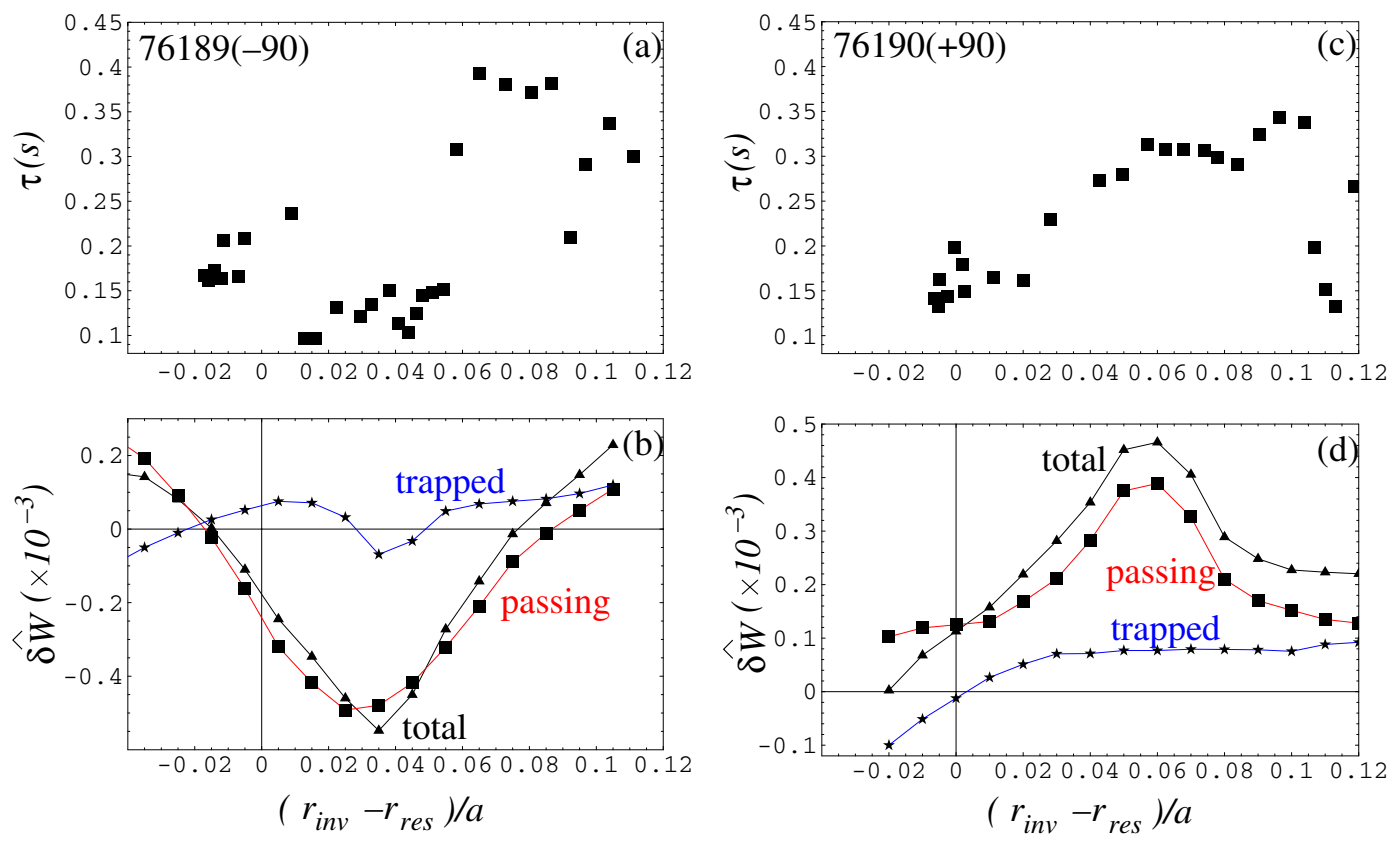

Figure 4. Showing $(a)$ and $(c)$ the sawtooth period for, respectively, pulses $76189\left(-90^{\circ}\right.$ phasing $)$ and $76190\left(+90^{\circ}\right.$ phasing $)$ plotted with respect to the difference between the smoothed average of the sawtooth inversion minor radius and the ${ }^{3} \mathrm{He}$ resonance minor radius. Plotted in $(b)$ and $(d)$ are the corresponding ICRH ion contributions to $\delta W$ assuming $r_{1}=r_{\text {inv }}$. (Colour online.)

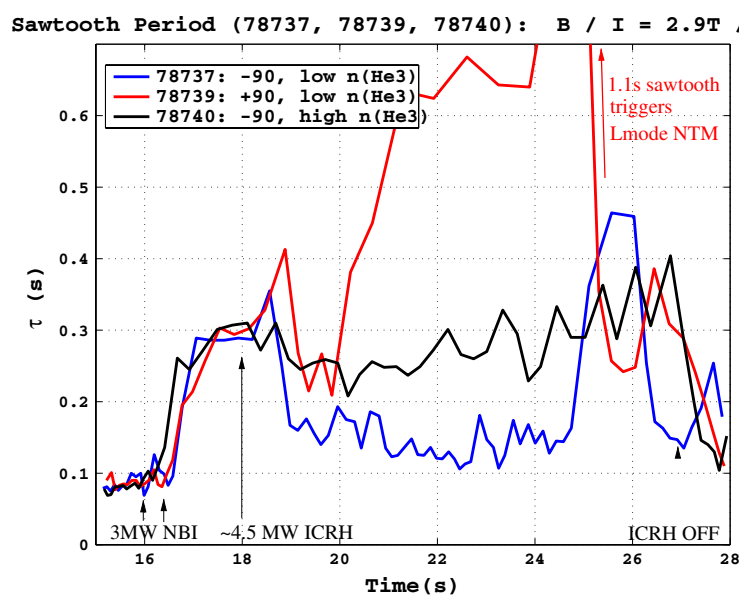

Figure 5. The sawtooth period for $78737\left(-90^{\circ}\right.$ phasing, low concentration $\left.{ }^{3} \mathrm{He}\right), 78740\left(-90^{\circ}\right.$ phasing, high concentration $\left.{ }^{3} \mathrm{He}\right)$ and $78739\left(+90^{\circ}\right.$ phasing, shown also in figure 1$)$.

as $\Delta_{r} \propto T_{\mathrm{h}}^{1 / 2}$. Referring e.g. to Stix [22], the hot ion temperature is proportional to the ICRH power, and inversely proportional to the minority ion density. Keeping the ICRH power constant ensures that the well-known stabilizing kinetic effects [3] (proportional to the trapped ion pressure $\approx n_{\mathrm{h}} T_{\mathrm{h}}$ profile inside $q=1$ ) are not strongly modified from pulse to pulse over a range of ${ }^{3} \mathrm{He}$ concentrations far below mode conversion, and hence in addition prevents significant current drive. Pulse 78740 shown in figure 5 employs approximately $4.5 \mathrm{MW}$ of $-90^{\circ}$ phasing ICRH with relatively high minority ${ }^{3} \mathrm{He}$ concentration (up to $3 \%$ of the electron density). This can be compared directly with the otherwise identical pulse 78737 , detailed also in figure 1 , employing $-90^{\circ}$ phasing with relatively low minority ${ }^{3} \mathrm{He}$ concentration (up to $n_{\mathrm{h}} / n_{\mathrm{e}}=$

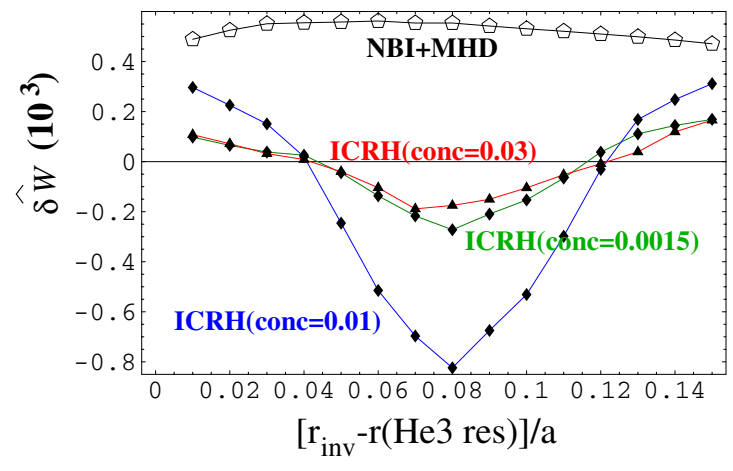

Figure 6. Stability $(\delta \hat{W})$ calculations, plotted with respect to $r_{1}-r_{\text {res }}$. Curves with $n_{\mathrm{h}} / n_{\mathrm{e}}=0.01$ and $n_{\mathrm{h}}=0.03$ correspond approximately to the conditions of 78737 and 78740 , respectively. The NBI and MHD contributions are also shown.

$0.6 \%$ ). The several-fold increase in ${ }^{3} \mathrm{He}$ concentration in 78740 , relative to 78737 , is consistent with the deliberate increased opening of the ${ }^{3} \mathrm{He}$ gas valve. The two pulses exhibit the same signature with respect to the scan in resonance position, but the amplitude of the effect is reduced for increased concentration, as expected for the fast ion mechanism, and contrary to the current drive mechanism [9] (currents remain negligible).

Detailed verification that the fast ion mechanism [6] is consistent with the experiments shown in figure 5 is undertaken by SELFO/HAGIS simulations evaluating the stability of JET pulses 78737 and 78740. Figure 6 plots the ICRH ion contribution to $\delta \hat{W}$, upon variation of $r_{1}-r_{\text {res }}$, for $n_{\mathrm{h}} / n_{\mathrm{e}}=0.01$ and $n_{\mathrm{h}} / n_{\mathrm{e}}=0.03$, relevant for 78737 and 78740 , respectively. It is seen that the range in $r_{1}-r_{\text {res }}$ over which ICRH has a destabilizing effect is independent of concentration. However, the strength of destabilization of 
counter propagating ICRH waves on the internal kink mode is more sensitive to concentration than would be expected from the simple relation $\Delta_{r} \propto\left(n_{\mathrm{e}} / n_{\mathrm{h}}\right)^{1 / 2}$. For $n_{\mathrm{h}} / n_{\mathrm{e}}=0.01$ the effect of ICRH dominates equation (1), while for $n_{\mathrm{h}} / n_{\mathrm{e}}=0.03$ the effect of ICRH is much smaller than the combined effect of NBI and MHD, as expected from experiments (figure 5). Finally, if the ${ }^{3} \mathrm{He}$ concentration is too low, minority power absorption is reduced, and enhanced minority ion energies lead to broader hot ion deposition, and losses, and a reduced impact on sawteeth, as indicated by the simulation in figure 6 employing $n_{\mathrm{h}} / n_{\mathrm{e}}=0.0015$.

This letter verifies that the kinetic response of highly energetic ions on the internal kink mode, described in [6], is sufficient to explain highly effective sawtooth control techniques (e.g. $[9,10]$ ) by toroidally propagating ICRF waves with resonance tangential to the $q=1$ surface. This has been achieved by creating experiments capable of eliminating all other known control mechanisms. Furthermore, more advanced experimental verification was undertaken by variation of the amplitude of the analytically derived fast ion mechanism. That fast ions can so dramatically, and directly, affect sawteeth is encouraging for ITER, especially where control solely via the magnetic shear is expected to be more challenging.

This work, supported by the Swiss National Science Foundation and by the European Communities under contract of Association between EURATOM and Confédération Suisse, was carried out within the framework of the European Fusion Development Agreement. The views and opinions expressed herein do not necessarily reflect those of the European Commission.

Euratom @ 2010

\section{References}

[1] Campbell D.J. et al 1988 Phys. Rev. Lett. 602148

[2] Phillips C.K. et al 1992 Phys. Fluids B 42155

[3] White R.B. et al 1988 Phys. Rev. Lett. 602038

[4] von Goeler S., Stodiek W. and Sauthoff N. 1974 Phys. Rev. Lett. 331201

[5] Sauter O. et al 2002 Phys. Rev. Lett. 88105001

[6] Graves J.P., Chapman I.T., Coda S., Eriksson L.-G. and Johnson T. 2009 Phys. Rev. Lett. 102065005

[7] Graves J.P. 2004 Phys. Rev. Lett. 92185003

[8] Chapman I.T. et al 2008 Plasma Phys. Control. Fusion 50045006

[9] Bhatnagar V.P. et al 1994 Nucl. Fusion 341579

[10] Eriksson L.-G. et al 2004 Phys. Rev. Lett. 92235004

[11] Fisch N.J. et al 1987 Rev. Mod. Phys. 59175

[12] Laxåback M. and Hellsten T. 2005 Nucl. Fusion 451510

[13] Parisot A. et al 2007 Plasma Phys. Control. Fusion 49219

[14] ITER Physics Basis Editors 1999 Nucl. Fusion 392137

[15] Carrera R. et al 1986 Phys. Fluids 29899

[16] Porcelli F., Boucher D. and Rosenbluth M.N. 1996 Plasma Phys. Control. Fusion 382163

[17] Angioni C. et al 2003 Nucl. Fusion 43455

[18] Lennholm M. 2009 Phys. Rev. Lett. 102115004

[19] Hedin J. et al 2002 Nucl. Fusion 42527

[20] Hellsten T., Carlsson J. and Eriksson L.-G. 1995 Phys. Rev. Lett. 743612

[21] Pinches S.D. et al 1998 Comput. Phys. Commun. 111133

[22] Stix T.H. 1975 Nucl. Fusion 15737 